\title{
REDUCTIVE ALGEBRAS OF COMPACT OPERATORS ${ }^{1}$
}

\author{
ROBERT A. BEKES
}

ABSTRACT. A closed subalgebra 2 of the bounded operators on a Hilbert space is called reductive if every closed invariant subspace for 2 is reducing for $थ$. We show that every reductive subalgebra of the compact operators is selfadjoint.

Introduction. Let $H$ be a Hilbert space. Let $B(H)$ denote the algebra of bounded operators on $H, K(H)$ the algebra of compact operators on $H$ and $F(H)$ the algebra of bounded operators on $H$ with finite dimensional range. A closed subalgebra $\mathfrak{U}$ of $B(H)$ is called reductive if every closed invariant subspace for $\mathscr{U}$ is reducing for $\mathscr{U}$ [i.e. if $M$ is a closed invariant subspace for $\mathscr{U}$ then $M$ is also invariant for $\left.\mathfrak{U}^{*}=\left\{T^{*}: \quad T \in \mathfrak{U}\right\}\right]$.

It is not known whether every weakly closed reductive algebra is selfadjoint. Using Lomonosov's theorem [5], H. Radjavi and P. Rosenthal have shown that every reductive subalgebra of $K(H)$ is selfadjoint [7]. This generalizes the following earlier result due to the author [4]: Every semisimple reductive subalgebra of the compact operators is selfadjoint.

In this paper we will show that every reductive subalgebra of $K(H)$ is semisimple and then prove Radjavi and Rosenthal's result using our techniques.

We introduce some notation. Let $\mathscr{U} \subset B(H)$ and $M \subset H$. Then cl( $\mathscr{U})$ denotes the norm closure of $\mathscr{Q}$ in $B(H)$ and $\mathrm{cl}(M)$ denotes the closure of $M$ in $H$. $\mathfrak{U}_{M}$ denotes the span of $\{T x: T \in \mathfrak{U}$ and $x \in M\}$. If $M$ is a closed invariant subspace for $T \in B(H)$ then $T \mid M$ denotes the operator $T$ restricted to $M$. If $M$ is a closed invariant subspace for $\mathscr{U} \subset B(H)$ then $\mathfrak{U} \mid M=\{T \in B(M): T=$ $S \mid M$ for some $S \in \mathfrak{O}\}$.

The main result. Let $X$ be a Banach space. A subalgebra of $B(X)$, the bounded operators on $X$, is called transitive or irreducible if it has noproper

Received by the editors February 11, 1974 .

AMS (MOS) subject classifications (1970). Primary 47C05; Secondary 47B05.

Key words and phrases. Algebra of operators, compact operators, invariant subspace, reducing subspaces, selfadjoint algebra.

${ }^{1}$ This research constitutes part of the author's Ph.D. thesis written at the University of Oregon under the direction of Professor B. A. Barnes.

Copyright (c) 1975. American Mathematical Society 
closed invariant subspaces. The following version of Lomonosov's theorem will be used. See [5, Lemma 1$]$.

Lemma 1. Let $\mathfrak{Q}$ be a transitive subalgebra of $B(X)$ and $T$ a nonzero compact operator on $X$. Then there exist $R \in \mathfrak{U}$ and $x \in X, x \neq 0$, such that $R T x=x$.

We will also need the next result, which is contained in the proof of [6, Theorem 3].

Lemma 2. Let $\mathfrak{A}$ be a reductive subalgebra of $B(H)$ and $T$ a nonzero compact operator in 2 . Then there exists a closed reducing subspace $M$ for थ such that $T \neq 0$ on $M$ and $\mathscr{U}$ acts transitively on $M$.

From these two lemmas we get

Lemma 3. Let $\mathfrak{Q}$ be a reductive subalgebra of $K(H)$. Then $\mathfrak{A}$ is semisimple.

Proof. Suppose $T$ is in the radical of $\mathfrak{X}$. If $T \neq 0$ then by Lemma 2 there exists a closed reducing subspace $M$ for $\mathscr{U}$ such that $T \neq 0$ on $M$ and $\mathscr{U}$ acts transitively on $M$. By Lemma 1 there exist $R \in \mathfrak{U}$ and $x \in M, x \neq 0$, such that $R T x=x$. This implies that 1 is in the spectrum of $R T$ when computed in $\mathscr{U}$. But $R T$ is in the radical of $\mathscr{U}$. This is a contradiction. Hence $T=0$ and therefore $\mathfrak{U}$ is semisimple.

In the next lemma we determine the structure of the minimal closed twosided ideals of a reductive subalgebra of $K(H)$. These ideals turn out to be the basic building blocks for reductive algebras of compact operators. An element $E$ of a complex normed algebra $\mathscr{Q}$ is called a minimal idempotent of $\mathfrak{A}$ if $E^{2}=E$ and $E^{\mathfrak{Q}} E=\{\lambda E: \lambda$ is complex $\}$. If $x, y \in H$ then the operator $x \otimes y$ is defined by $x \otimes y(z)=(z, y) x$ for all $z \in H$. Then $\|x \otimes y\|=$ $\|x\|\|y\|$.

Lemma 4. Let 2 be a reductive subalgebra of $K(H)$ and $E \neq 0$ a minimal idempotent of $\mathfrak{X}$. Then $\mathrm{cl}(\mathfrak{U E} \mathscr{U})$ is selfadjoint and isomorphic to $\mathfrak{A} \mid \mathrm{cl}(\mathfrak{Q} E H)$.

Proof. By induction choose $x_{1}, \ldots, x_{k} \in H$ such that $E x_{i}=x_{i}$, $\left\|x_{i}\right\|=1$ for $i=1, \cdots, k$ and $\mathfrak{U}_{x_{i}} \perp \mathfrak{A}_{x_{j}}$ for $i \neq j$. If $E$ is zero on $\left(\mathscr{U}_{x_{1}} \oplus \ldots \oplus \mathscr{U}_{x_{k}}\right)^{\perp}$, then the induction stops. If $E$ is not zero on $\left(\mathscr{U} x_{1} \oplus \ldots \oplus \mathscr{U} x_{k}\right)^{\perp}$, then choose $z \in\left(\mathscr{V} x_{1} \oplus \ldots \oplus \mathscr{U} x_{k}\right)^{\perp}$ such that $E z \neq 0$. Set $x_{k+1}=E z\|E z\|^{-1}$. Then $E x_{k+1}=x_{k+1}$ and $\left\|x_{k+1}\right\|=1$. Also, since $\mathfrak{U}$ is reductive, $2 x_{k+1} \perp \mathfrak{2} x_{j}$ for $j=1, \ldots, k$. This process must stop in a 
finite number of steps since $E$ has finite dimensional range. Suppose it stops in $n$ steps. By [2, Lemma 1] for each $i=1, \cdots, n$ there exists $y_{i} \epsilon$ $\operatorname{cl}\left(\mathscr{U}_{x_{i}}\right)$ such that $\left(x_{i}, y_{i}\right)=1$ and $E \mid \operatorname{cl}\left(\mathscr{U} x_{i}\right)=x_{i} \otimes y_{i}$. Now since the $\mathscr{U}_{x_{i}}$ are orthogonal and $E=0$ on $\left(\mathfrak{U}_{x_{1}} \oplus \ldots \oplus \mathfrak{U}_{x_{n}}\right)^{\perp}$ we have

$$
E=\sum_{i=1}^{n} x_{i} \otimes y_{i}
$$

Set $x=x_{1}+\cdots+x_{n}$. Then again by [2, Lemma 1] there exists $w \in \operatorname{cl}(2 x)$ such that $E \mid \operatorname{cl}(\mathscr{R} x)=x \otimes w$.

We claim that $\mathscr{P l}_{x}$ is closed in $H$. To see this suppose that $\left\{T_{i} x\right\}_{i=1}^{\infty}$ is a Cauchy sequence in $\mathfrak{U}_{x}$. Let $s_{1}, \ldots, S_{n} \in \mathscr{U}$. Then

$$
\begin{aligned}
\left\|\left(T_{i} E-T_{j} E\right)\left(S_{1} x_{1}+\cdots+S_{n} x_{n}\right)\right\|=\left\|\sum_{k=1}^{n}\left(S_{k} x_{k}, y_{k}\right)\left(T_{i} x_{k}-T_{j} x_{k}\right)\right\| \\
\leq \sum_{k=1}^{n}\left\|S_{k} x_{k}\right\|\left\|y_{k}\right\|\left\|T_{i} x_{k}-T_{j} x_{k}\right\| \\
\leq \sum_{k=1}^{n}\left\|S_{1} x_{1}+\cdots+S_{n} x_{n}\right\|\left\|y_{1}+\cdots+y_{n}\right\|\left\|T_{i} x-T_{j} x\right\| \\
=n\left\|S_{1} x_{1}+\cdots+S_{n} x_{n}\right\|\left\|y_{1}+\cdots+y_{n}\right\|\left\|T_{i} x-T_{j} x\right\| .
\end{aligned}
$$

Since $E=0$ on $\left(\mathscr{U}_{x_{1}} \oplus \ldots \oplus \mathscr{U} x_{n}\right)^{\perp},\left\{T_{i} E\right\}_{i=1}^{\infty}$ is a Cauchy sequence in $\mathscr{U} E$, which is closed in $\mathfrak{U}$. So there exists $S \in \mathfrak{U}$ such that $\left\|T_{i} E-S E\right\| \rightarrow 0$. Therefore $\left\|T_{i} x-S x\right\| \rightarrow 0$ and so $\mathscr{U}_{x}$ is closed in $H$.

Now $E^{*}(x)=\sum_{i=1}^{n} y_{i} \otimes x_{i}(x)=y_{1}+\cdots+y_{n}$. But since $\mathscr{O}$ is reductive, $E^{*} \mid \mathscr{Q}_{x}=w \otimes x$. So we also have $E^{*}(x)=w \otimes x(x)=\|x\|^{2} w$. If we set $y=y_{1}$ $+\cdots+y_{n}$, then $y=\|x\|^{2} w=n w \in \mathscr{U} x$. By Lemma 3, $\mathcal{U}$ is semisimple and so semiprime. Therefore by the proof of [2, Lemma 2] $\mathscr{O}$ acts algebraically irreducibly on $\mathscr{U} x$. So there exists $R \in \mathcal{U}$ such that $R y=x$. Then $R y_{i}=x_{i}$ for $i=1, \cdots, n$. Now let $T \in \mathcal{U}$. Then

$$
\begin{aligned}
E R^{*} T^{*} T x & =x \otimes w\left(R^{*} T^{*} T x\right)=\left(R^{*} T^{*} T x, w\right) x \\
& =n^{-1}(T x, T x) x=\sum_{i=1}^{n} n^{-1}\|T x\|^{2} x_{i}
\end{aligned}
$$

But we also have

\section{Therefore}

$$
E R^{*} T^{*} T x=\sum_{i=1}^{n} x_{i} \otimes y_{i}\left(R^{*} T^{*} T x\right)=\sum_{i=1}^{n}\left(R^{*} T^{*} T x_{i}, y_{i}\right) x_{i}=\sum_{i=1}^{n}\left\|T x_{i}\right\|^{2} x_{i}
$$




$$
n^{-1 / 2}\|T x\|=\left\|T x_{i}\right\| \text { for all } T \in \text { ?l }
$$

and $i=1, \cdots, n$. From this set of equalities it follows that each of the spaces $\mathfrak{U}_{x_{i}}$ is bicontinuously isomorphic to $\mathfrak{U}_{x}$. In particular each $\mathfrak{U}_{x_{i}}$ is closed in $H$ and $\mathfrak{A} x_{i}$ is isometrically isomorphic to $\mathfrak{U} x_{j}$ for all $i$ and $j$. So for all $T \in \mathfrak{Q}$ we have

$$
\left\|T\left|\mathfrak{M} x_{i}\|=\| T\right| \Re x_{j}\right\| .
$$

Now since $\mathfrak{U}$ is reductive, $\mathrm{cl}\left(\mathfrak{U}^{*} y_{i}\right)$ is an invariant subspace for $\mathfrak{U}$. And since $\mathfrak{U}$ acts irreducibly on $\mathfrak{U}_{x_{i}}$ and $E^{*} y_{i}=y_{i} \neq 0$, we have $\operatorname{cl}\left(\mathscr{U} * y_{i}\right)=\mathfrak{U}_{x_{i}}$. Therefore

$$
F\left(\mathfrak{L C} x_{i}\right) \subset \mathrm{cl} \mathrm{span}\left\{S x_{i} \otimes T^{*} y_{i} \mid \mathfrak{Q} x_{i}: S, T \in \mathfrak{Y}\right\} .
$$

But $S x_{i} \otimes T^{*} y_{i}\left|\mathscr{U} x_{i}=S E T\right| \mathscr{U} x_{i}$. Therefore

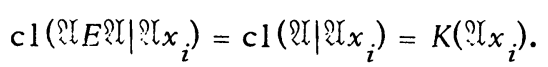

Now the $\mathscr{U} x_{i}$ are orthogonal and reduce $\mathscr{U}$, and $\mathscr{U} E \mathscr{U}=\{0\}$ on $\left(\mathscr{U} x_{1} \oplus \cdots \oplus \mathscr{U} x_{n}\right)^{\perp}$, so for $T \in \operatorname{cl}(\mathscr{U} E \mathscr{U})$ and each $i$ we have

$$
\|T\|=\left\|T\left|\mathfrak{T E} H\left\|=\max \left\{\left\|T \mid ? x_{j}\right\|: j=1, \cdots, n\right\}=\right\| T\right| \mathfrak{T} x_{i}\right\| \cdot
$$

Therefore $\operatorname{cl}(\mathfrak{U} E \mathscr{U}) \mid \mathfrak{U}_{x_{i}}$ is closed in $K\left(\mathfrak{U}_{x_{i}}\right)$, and so

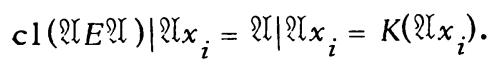

Now let $T \in \operatorname{cl}(\mathscr{U} E \mathscr{U})$. Then since $\mathscr{U}$ is reductive, by (3) we have $T^{*} \mid \mathfrak{A}_{x_{i}}=$ $\left(T \mid \mathscr{U}_{x_{i}}\right)^{*} \in \operatorname{cl}\left(\mathscr{U}_{E} \mathfrak{U}\right) \mid \mathscr{U}_{x_{i}}$. Also $T^{*} \mid\left(\mathscr{U}_{E H}\right)^{\perp}=\left(T \mid\left(\mathscr{U}_{E H}\right)^{\perp}\right)^{*}=0$. So (1) and (2) imply that $T^{*} \in \mathrm{cl}\left(\mathfrak{U}_{E} \mathfrak{U}\right)$. Hence $\mathrm{cl}\left(\mathscr{U}_{E} \mathfrak{U}\right)$ is selfadjoint and isomorphic to थl $\mid \mathrm{cl}(\mathfrak{U E H})$.

Theorem. Let $\mathfrak{U}$ be a reductive subalgebra of $K(H)$. Then $\mathfrak{U}$ is selfadjoint.

Proof. Let $\mathcal{I}$ be a maximal set of minimal idempotents of $\mathscr{U}$ such that if $E_{1}, E_{2} \in \mathfrak{I}$ with $E_{1} \neq E_{2}$ then $\mathrm{cl}\left(\mathfrak{U} E_{1} \mathfrak{U}\right) \neq \mathrm{cl}\left(\mathfrak{U}_{E_{2}} \mathfrak{U}\right)$. We complete the proof in three steps.

I. We first prove that if $E_{1}, E_{2} \in \mathfrak{U}$ with $E_{1} \neq E_{2}$ then $\mathfrak{U}_{E_{1}} H \perp \mathfrak{U}_{E_{2}} H$. Let $T \in \mathfrak{A}$. Then

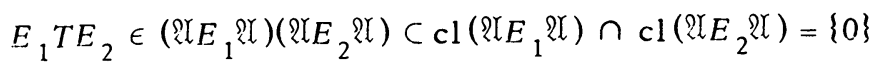

since the $\operatorname{cl}\left(\mathscr{U}_{E_{i}} \mathscr{U}\right)$ are distinct minimal closed ideals $[1, \mathrm{p} .335]$. Since $\mathscr{U}$ is reductive,

$$
E_{1}^{*} \mid \mathfrak{L} E_{2} H=\left(E_{1} \mid \mathfrak{N} E_{2} H\right)^{*}=0 \text { and } \mathfrak{R} *\left(\mathfrak{S} E_{2} H\right) \subset \mathrm{cl}\left(\mathfrak{Q} E_{2} H\right) \text {. }
$$


Now let $R, S \in \mathscr{U}$ and $u, v \in H$. Then $\left(R E_{1} u, S E_{2} v\right)=\left(u, E_{1}^{*} R^{*} S E_{2} v\right)=0$, which completes step I.

II. Let $M=$ closed $\operatorname{span}\left\{\mathfrak{U}_{E H}: E \in \mathfrak{I}\right\}$. Then

$$
\mathfrak{N} \mid M=\operatorname{cl}\left(\sum_{E \in \mathcal{g}} \oplus \mathfrak{Q} E \mathfrak{R}\right) M \quad \text { and } \mathfrak{Y} \mid M=\{T \mid M: T(M)=\{0\}\} \text {. }
$$

To see this let $T \in \mathscr{U}$ and $\epsilon>0$. Since $T$ is compact and the $\mathscr{Y} E H$ are orthogonal and reducing for $\mathscr{U}$, we must have $\{E \in \mathscr{I}:\|T \mid \mathfrak{U} E H\| \geq \epsilon\}$ a finite set, say $\left\{E_{1}, \cdots, E_{n}\right\}$. By Lemma 4 choose $T_{i} \in \mathrm{cl}\left(\mathfrak{U}_{E_{i}} \mathfrak{U}\right)$ such that $T\left|\operatorname{cl}\left(\mathfrak{U}_{E_{i} H}\right)=T_{i}\right| \operatorname{cl}\left(\mathfrak{U}_{E_{i}} H\right)$ for $i=1, \cdots, n$. Set $T_{0}=\sum_{i=1}^{n} T_{i}$. Then

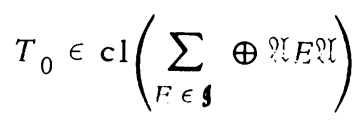

and

$$
\left\|\left(T-T_{0}\right) \mid M\right\|=\max \left\{\| T \mid M\left(E H \|: E \in \mathbb{A} \backslash\left\{E_{1}, \cdots, Z_{n}\right\}\right\} \leq \epsilon .\right.
$$

Therefore $T\left|M \in \operatorname{cl}\left(\Sigma_{E \in g} \oplus \mathfrak{U} E \mathscr{U}\right)\right| M$. Since $\operatorname{cl}\left(\Sigma_{E \in \mathfrak{g}} \oplus \mathfrak{U} E \mathscr{U}\right) \mid M=\{0\}$, it follows that $\mathscr{Q} \mid M^{\perp}=\left\{T \mid M^{\perp}: T(M)=\{0\}\right\}$.

III. We show that $M^{\perp}=\{0\}$ or $\mathscr{2} \mid M^{\perp}=\{0\}$. Suppose $\mathscr{N} \mid M^{\perp} \neq\{0\}$. Then by step II, we have that $\mathfrak{X} \mid M^{\perp}$ is a reductive subalgebra of $K\left(M^{\perp}\right)$, and so by Lemma 2 it is semisimple. Therefore there exists $T \in \mathfrak{U} \mid M^{\perp}$ such that the spectrum of $T$ in $\mathfrak{U} \mid M^{\perp}$ is not zero. We can produce a nonzero idempotent $E^{\prime}$ in $\mathscr{X} \mid M^{\perp}$ by taking the appropriate contour integral about a nonzero (isolated) point of the spectrum of $T . E^{\prime}$ must have finite dimensional range. But then by the proof of [3, Theorem 2] $\mathscr{U} \mid M^{\perp}$ contains a minimal idempotent. Also by claim 2 we may assume that this minimal idempotent is of the form $E_{0} \mid M^{\perp}$, where $E_{0} \in \mathfrak{O}$ and $E_{0}(M)=\{0\}$. Now since $\mathfrak{U}$ is reductive, $E_{0} T E_{0}$ $=0$ for all $T \in \mathscr{U}$ such that $T\left(M^{\perp}\right)=\{0\}$. Therefore $E_{0} \mathfrak{U}_{E_{0}}$ is isomorphic to $E_{0}\left(\mathfrak{U} \mid M^{\perp}\right) E_{0}=\left\{\lambda E_{0}: \lambda\right.$ is complex $\}$. So $E_{0}$ is a minimal idempotent of $\mathscr{U}$. But $\mathrm{cl}(\mathfrak{U} E \mathfrak{U}) \mid M=\{0\}$ for all $E \in \mathfrak{Y}$. So $\mathrm{cl}(\mathfrak{U} E \mathfrak{U}) \neq \mathrm{cl}\left(\mathfrak{U} E_{0} \mathfrak{U}\right)$ for all $E \in \mathfrak{Y}$. This violates the maximality of the set $\mathscr{I}$. Hence $\mathfrak{U} \mid M^{\perp}=\{0\}$.

Therefore $\mathscr{U}=\operatorname{cl}\left(\Sigma_{E \in \mathscr{g}} \bigoplus \mathfrak{U} E \mathscr{U}\right)$, and by Lemma 4 this implies that $\mathscr{U}$ is selfadjoint.

\section{REFERENCES}

1. B. A. Barnes, Lectures on Banach algebras, mimeographed notes, University of Oregon, Eugene, Ore., 1969-70.

2. Irreducible algebras of operators which contain a minimal idempotent, Proc. Amer. Math. Soc. 30 (1971), 337-342. MR 44 \#7303.

3. - Density theorems for algebras of operators and annihilator Banach algebras, Michigan Math. J. 19 (1972), 149-155. MR 46 \#6058. 
4. R. A. Bekes, Conditions under which an algebra containing a compact opera. tor is selfadjoint, Notices Amer. Math. Soc. 20 (1973), A-145. Abstract \#70 1-46-24.

5. C. Pearcy and A. Shields, $A$ survey of some results on invariant subspaces of operators on a Banach space (preprint).

6. H. Radjavi and P. Rosenthal, A sufficient condition that an operator algebra be self-adjoint, Canad. J. Math 23 (1971), 588-597.

7. - On transitive and reductive operator algebras (preprint).

DEPARTMENT OF MATHEMATICS, ARIZONA STATE UNIVERSITY, TEMPE, ARIZONA 85281

Current address: Department of Mathematics, Dartmouth College, Hanover, New Hampshire 03755 